\title{
The microscope drape method to reduce aerosolisation during endoscopic sinus and skull base surgery in the COVID era. How i do it
}

\author{
Aikaterini Tsagkovits $^{1}\left[\right.$ D $\cdot$ Dimitrios loannidis $^{1} \cdot$ Ashok Rokade $^{1}$
}

Received: 12 September 2020 / Accepted: 13 October 2020 / Published online: 28 October 2020

(c) Springer-Verlag GmbH Germany, part of Springer Nature 2020

\begin{abstract}
Background Otolaryngologists are faced with concerning challenges since the onset of the coronavirus disease (COVID-19) pandemic due to significant risk of occupational infection. Transmission can happen during intraoperative exposure to viral particles carried by droplets or aerosols. Endoscopic sinus and skull base surgery are notable for causing aerosolisation, putting healthcare staff at substantial risk.

Method We describe the creation of a tight-seal tent from a microscope drape covering the operative field and the operator's hands with the aim to contain aerosols during transnasal endoscopic surgery.

Conclusion The microscope drape technique is a simple barrier measure that could potentially improve safety during endoscopic procedures.
\end{abstract}

Keywords Microscope drape · COVID-19 · Endoscopic sinus surgery $\cdot$ FESS

\section{Background}

The 2019 novel coronavirus (SARS-CoV-2) outbreak poses significant risk to healthcare professionals during the provision of clinical care. Transmission can happen through respiratory droplets and aerosolised viral particles, which can be produced during certain airway procedures, such as intubation and airway suctioning [1,2]. There have been anecdotal reports of increased number of coronavirus disease (COVID-19) cases among otolaryngologists worldwide [3]. Endoscopic sinus and skull base surgery are considered high-risk operations for viral shedding and subsequent infection of the operator and the scrub team. This is due to the high viral load in the upper airway of the infected patient, especially in the nose and the nasopharynx, and the aerosol generation that can happen during endonasal instrumentation, such as suctioning, bone drilling and use of microdebrider and bipolar diathermy [4]. Despite the use of enhanced personal protective equipment, there are concerns

Aikaterini Tsagkovits tsagkovits@yahoo.gr

1 ENT Department, Royal Hampshire County Hospital, Romsey Rd, Winchester SO22 5DG, UK that SARS-CoV-2 could still be contacted when spread at the wider theatre environment, where it can remain for up to $3 \mathrm{~h}$ in aerosolised form [1]. This highlights the need for development and implementation of strategies to minimise aerosol spread in the form of barrier cover, and thus reduce viral exposure during these endoscopic procedures. Here, we present a potential solution to contain the circulating aerosols produced during functional endoscopic sinus surgery (FESS) in the operating room by the creation of a tent with a surgical otomicroscope drape.

\section{Methods}

After general anaesthesia and standard draping, personal protective equipment (PPE) is worn by the surgical team as per guidance. A magnetic mat with the instruments that are expected to be used is placed on the patient's thorax. An anaesthesia screen (Eshmann Technologies Ltd.@) is placed on the patient's left side at the level of the midsternum (Fig. 1). An otomicroscope drape (Microtek ${ }^{\mathrm{TM}}$ Microscope Mini Drape) is wrapped around the patient's head and upper torso over the screen and tucked under the surgical bed. Slits are cut at an ergonomic position and the surgeon's arms are inserted within the tent (Fig. 2). A third slit is made 


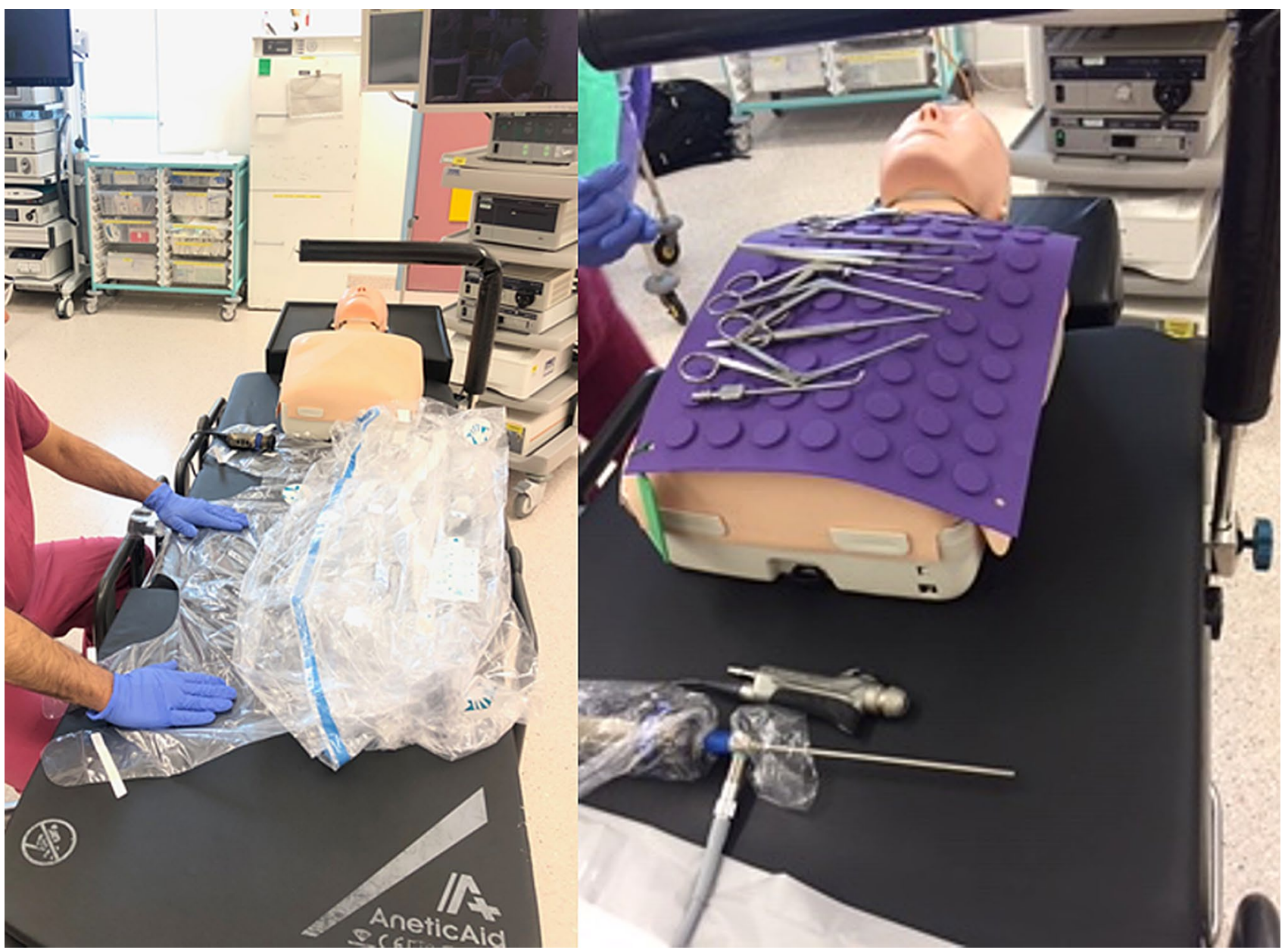

Fig. 1 Equipment for the surgical tent: otomicroscope drape, anaesthesia screen (left) and magnetic mat (right)

to introduce the endoscopic camera, light source, bipolar, endoscrub and suction cables. All slits are sealed with sterile tape. During the procedure, instruments can be handed over by an assistant from outside of the tent or picked up by the surgeon. At the end of the surgery, doffing is accomplished with the help of an assistant and the microscope drape can be removed at the same time.

The method was simulated and tested in the operating room with an adult airway manikin before its first application, where the pictures on the figures were taken from. Expert advice was sought by the trust's research department and special approval and consent were not deemed necessary as the intervention was based on best public health evidence and did not deviate from the standard surgical technique.

The surgical tent was used for an urgent examination of the nose under anaesthesia and nasopharyngeal biopsy in an 11-year-old child with a postnasal tumour with unknown COVID-19 status. The procedure was accomplished comfortably without any compromise of vision or hand movements on the surgical field. The patient had an uneventful recovery. Neither the surgeon or any of the healthcare professionals involved in the procedure tested positive for COVID-19 within 14 days of the surgery.

\section{Discussion}

We suggest a simple mitigation strategy for containing aerosol generation during endoscopic sinus surgery with readily available equipment in every ENT theatre. The sensitivity of real-time reverse transcription PCR from nasopharyngeal and oropharyngeal swab specimens, which is the current practice for preoperative screening, ranges between 63 and $73 \%[5,6]$, rendering the viral detection in surgical patients dubious. Additionally, asymptomatic and pre-symptomatic SARS-CoV-2 transmission mandate precautionary measures like that to reduce viral spread [7]. Similar barrier techniques were already proposed and successfully used in other airway procedures during the COVID era, highlighting the fact that ENT surgeons have appreciated the importance 
Fig. 2 Surgical tent setup during simulation

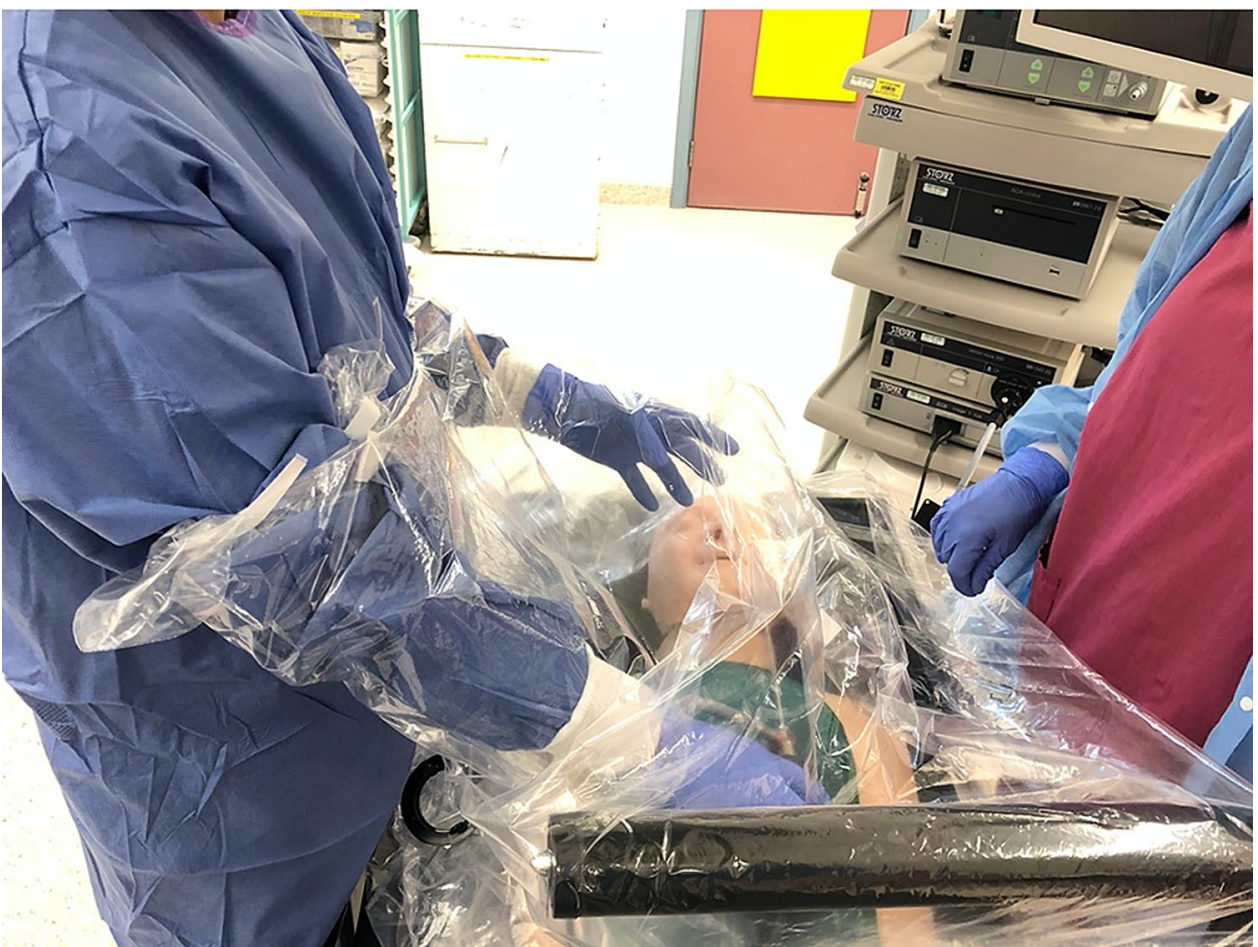

of aerosol mitigation during ENT procedures [8-10]. Our method allowed for unrestricted hand movements and there was not any interference in the visualisation of the surgical field e.g., glaring from the plastic transparent cover previously described [8]. Contrary to the covers with open slits or ports, our tight-seal arrangement around the cables and incorporating the surgeon's arms within the drape ensures minimal aerosol escape [8-10].

We acknowledge that our technique necessitates all the equipment required for the procedure to be placed on the patient's chest before the application of the drape. The instruments should be carefully chosen in advance and kept at a reasonable number as they are placed on a magnetic mat with dimensions $30 \times 40 \mathrm{~cm}$ (Purple Surgical (C). Extra temporary storage for instruments could be provided by instrument adhesive pouches secured on the patient's side. Uncovering the field during the procedure to hand in extra pieces would allow potential aerosol leak and should be avoided.

Additional time should be spared to prepare the surgical tent, which must be accounted for anaesthetic and logistic planning purposes. However, preparation time is expected to shorten after practising the technique a few times. Simulating the technique with a manikin is advisable before proceeding with a real patient. The microscope drape technique setup would certainly impact surgical training in endoscopic sinus surgery, as the initial operator should be able to perform the procedure until the end without being able to allocate surgical time to a trainee at any step, which is one of the limitations. The surgical tent is a new method that is evolving and further improvements are being designed. The addition of negative pressure by adding a second suction within the drape could offer additional safety. More sophisticated configurations with isolating chambers within the drape for instrument insertion from the outside without risking aerosol release could be considered. Further experiments with simulated aerosols to assess the effectiveness of the technique would be necessary.

Acknowledgements The authors would like to thank Mrs Hasnaa Ismail-Koch for her valuable feedback on the application of the technique. Due to unforeseen circumstances and unprecedented pressure in our specialty we felt the need to disseminate our technique to our colleagues for important safety reasons before submission. There is a preliminary version of the technique published on the ENTUK website: (https://www.entuk.org/sites/default/files/Safer\%20FESS\%20in\%20the $\% 20$ COVID $\% 20$ Era\%20for\%20ENTUK\%20.pdf)

Author contributions Conceptualization: AR; Methodology: AR, DI, AT; Writing—original draft preparation: AT; Writing—review and editing: AR, DI; Supervision: AR.

Funding No funding was received.

Code availability Not applicable.

\section{Compliance with ethical standards}

Conflicts of interest The authors declare that they have no conflict of interest. 
Ethics approval Ethical approval was waived by the hospital's research department in view of the non-interventional nature of the method performed on a procedure that was part of the routine care.

Consent to participate Not applicable.

Consent for publication Not applicable.

Availability of data and material Not applicable.

\section{References}

1. van Doremalen N, Bushmaker T, Morris DH et al (2020) Aerosol and surface stability of SARS-CoV-2 as compared with SARSCoV-1. N Engl J Med 382:1564-1567. https://doi.org/10.1056/ NEJMc2004973

2. Morawska L, Cao J (2020) Airborne transmission of SARSCoV-2: the world should face the reality. Environ Int 139:105730. https://doi.org/10.1016/j.envint.2020.105730

3. Jotz GP, Voegels RL, Bento RF (2020) Otorhinolaryngologists and Coronavirus Disease 2019 (COVID-19). Int Arch Otorhinolaryngol 24:e125-e128. https://doi.org/10.1055/s-0040-17097 24

4. Workman AD, Welling DB, Carter BS et al (2020) Endonasal instrumentation and aerosolization risk in the era of COVID-19: simulation literature review, and proposed mitigation strategies. Int Forum Allergy Rhinol. https://doi.org/10.1002/alr.22577

5. Wang W, Xu Y, Gao R et al (2020) Detection of SARS-CoV-2 in different types of clinical specimens. JAMA 323:1843-1844. https ://doi.org/10.1001/jama.2020.3786

6. Yang Y, Yang M, Shen C et al (2020) Evaluating the accuracy of different respiratory specimens in the laboratory diagnosis and monitoring the viral shedding of 2019-nCoV infections. medRxiv. https://doi.org/10.1101/2020.02.11.20021493

7. Wölfel R, Corman VM, Guggemos W et al (2020) Virological assessment of hospitalized patients with COVID-2019. Nature. https://doi.org/10.1038/s41586-020-2196-x

8. Bertroche JT, Pipkorn P, Zolkind P et al (2020) Negative-pressure aerosol cover for COVID-19 tracheostomy. JAMA Otolaryngol Neck Surg. https://doi.org/10.1001/jamaoto.2020.1081

9. Pollaers K, Herbert H, Vijayasekaran S (2020) Pediatric microlaryngoscopy and bronchoscopy in the COVID-19 Era. JAMA Otolaryngol Neck Surg. https://doi.org/10.1001/jamaoto.2020.1191

10. David AP, Jiam NT, Reither JM et al (2020) Endoscopic skull base and transoral surgery during COVID-19 pandemic: minimizing droplet spread with negative-pressure otolaryngology viral isolation drape. Head Neck. https://doi.org/10.1002/hed.26239

Publisher's Note Springer Nature remains neutral with regard to jurisdictional claims in published maps and institutional affiliations. 